Génét. Sél. Evol., 1983, 15 (2), 177-184

\title{
Mise en évidence d'une fission centromérique hétérozygote chez un mâle Theropithecus gelada et comparaison chromosomique avec les autres Papioninae
}

\author{
Martine MULERIS* B. DUTRILLAUX* et G. CHAUVIER** \\ avec la collaboration technique d'Anne-Marie DUTRILLAUX \\ * Institut de Progenèse, 15, rue de l'École de Médecine, F 75270 Paris cédex 06 \\ ** Museum national d'Histoire naturelle de Paris. \\ Laboratoire de la Ménagerie, 57, rue Cuvier, 75005 Paris
}

\begin{abstract}
Résumé
Le caryotype d'un mâle de Theropithecus gelada est décrit et comparé à celui des autres Papioni- nae (Cercopithecidae). Le caryotype normal de $T$. gelada comprend 42 chromosomes et est identique à celui des Papio, Lophocebus et Macaca. L'individu étudié ici est porteur hétérozygote d'une fission centromérique du chromosome 7. Le rôle des fissions chromosomiques dans l'évolution des Cercopithécoïdes est discuté.
\end{abstract}

Mots clés : Theropithecus - chromosome - fission - comparaison - Papioninae.

\section{Summary}

Demonstration of a heterozygote centromeric fission in a male

Theropithecus gelada and chromosomal comparison with the other Papioninae.

The karyotype of a Theropithecus gelada male specimen is described and compared to that of the other Papioninae (Cercopithecidae). Its normal karyotype is composed of 42 chromosomes and is similar to that of Papio, Lophocebus and Macaca. The specimen studied here is heterozygote for a centromeric fission of chromosome 7 . The role of chromosomal fissions in the evolution of Cercopithecoids is discussed.

Key words : Theropithecus - chromosome - fission - comparison - Papioninae.

L'étude systématique du caryotype de nombreuses espèces de Cercopithecoïdea a permis de reconstituer le caryotype probable de leur dernier ancêtre commun et d'établir leur phylogénie (DUTRILlaux et al., 1982). Le caryotype de Theropithecus gelada, qui n'avait pas été considéré pour cette étude est rapporté ici et comparé à celui des autres espèces de Papioninae. 


\section{Matériel et méthodes}

Le spécimen étudié provient de la ménagerie du Muséum National d'Histoire Naturelle de Paris, où il est gardé en captivité. Les cellules en mitose ont été obtenues après culture de lymphocytes à partir d'un prélèvement sanguin.

Diverses méthodes de marquage chromosomique ont été appliquées : bandes R (RHG et $\mathrm{RBG})$, bandes $\mathrm{Q}(\mathrm{QFQ})$, bandes $\mathrm{C}(\mathrm{CBG})$ et coloration des organisateurs nucléolaires (NOR).

\section{Résultats et discussion}

Le caryotype de l'animal étudié comprend 43 chromosomes, tous les autosomes étant appariables à l'exception de 3 éléments dont l'un, sub-métacentrique, correspond à deux acrocentriques (fig. 1). La description antérieure du caryotype de cette espèce rapportant une formule à 42 chromosomes (CHIARELLI, 1962) il est très probable que les deux acrocentriques non appariés dérivent par fission centrique du grand submétacentrique $n^{\circ} 7$. Un autre argument en faveur de la fission est qu'un chromosome semblable au chromosome 7 submétacentrique de $T$. gelada, équivalent des 14 et 15 humains, est retrouvé chez de nombreux primates et autres mammifères (observations personnelles). De plus, des gènes portés par les 14 et 15 humains sont retrouvés synténiques chez Microcebus murinus (prosimien) (COCHET et al., 1982) et chez le chat domestique (O'BRIEN et NASH, 1982), ce qui traduit l'origine très ancienne de ce chromosome.

Les différentes méthodes de marquage (bandes $R$, bandes $Q$, bandes $C$ ) ne montrent aucune particularité, et l'organisateur nucléolaire est situé sur la paire $\mathrm{n}^{\circ} 9$.

\section{A. Comparaison avec les autres Papioninae}

Les différentes espèces de Papioninae étudiées à ce jour possèdent toutes des chromosomes assez semblables (DuTRILlaux et al., 1982). Les espèces des genres Macaca (M. fascicularis excepté), Papio et Lophocebus possèdent le même caryotype, lui-même identique à celui de $T$.gelada (fig. 2). Celui-ci diffère du caryotype des espèces des genres Cercocebus et Mandrillus par un remaniement complexe affectant le chromosome 10. Ce nouveau résultat, qui porte à 4 le nombre de genres possédant le même caryotype confirme la notion de la grande stabilité du caryotype des Papioninae. Un autre argument en faveur de cette stabilité est la rareté des remaniements intraspécifiques. Ainsi, sur plus de 170 animaux dont le caryotype a été étudié avec marquage (BERNSTEIN et al., 1980, CAMBEFORT et al., 1976 a et b ; De VRIES et al., 1975 ; DutrillauX et al., 1978, 1979 a et b, 1982, SouliÉ et de GrOUCHY, 1981, STOCK et HSU, 1973) aucune anomalie structurale n'avait été jusqu'ici signalée. Le remaniement observé ici en serait donc un premier exemple.

\section{B. Rôle des fissions chromosomiques dans l'évolution des cercopithecoides}

Il est particulièrement intéressant que ce remaniement soit une fission car celles-ci sont, semble-t-il, très rares chez les primates, à l'exception des Cercopithecinae. Or les Papioninae forment une sous-famille proche, mais dans laquelle aucune fission n'est survenue au 


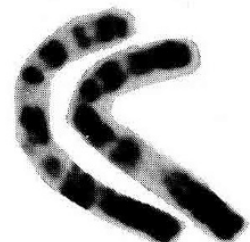

1

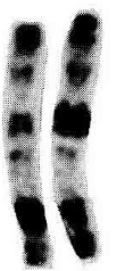

6

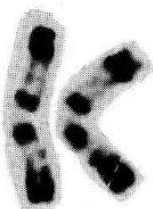

11

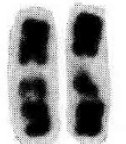

16

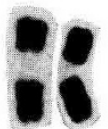

19

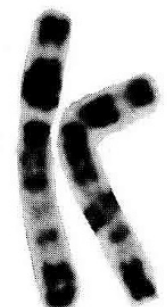

2

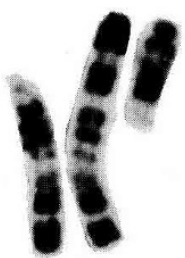

7

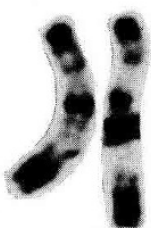

12

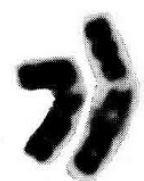

17

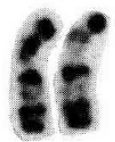

20

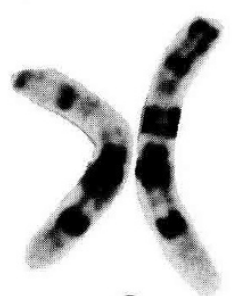

3

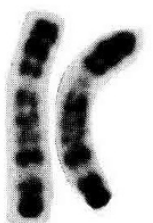

8

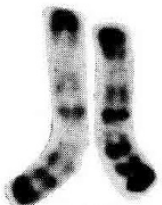

13

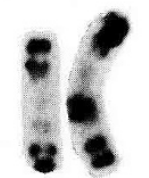

18

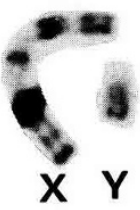

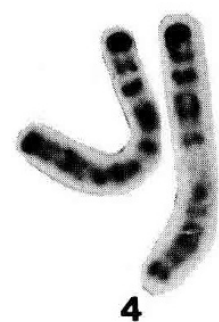

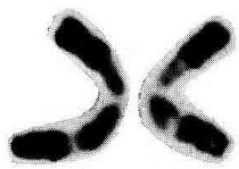

9

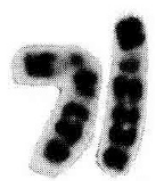

14

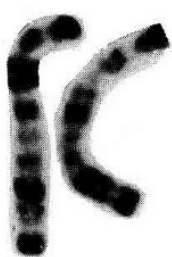

5

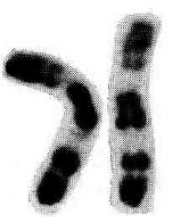

10

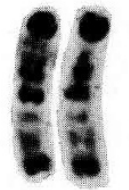

15

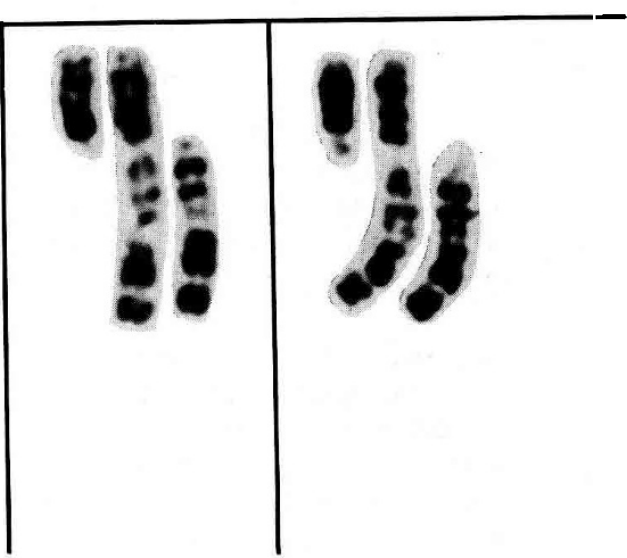

FIG. 1

Caryotype de Theropithecus gelada mâle, en bandes $R$ (RHG). On remarque la fission centromérique d'un chromosome 7 , représentée également en bandes $R B G$ dans l'encadré.

Karyotype of a male Theropithecus gelada after $R$-banding (RHG). Notice the existence of a centromeric fission of one of the chromosomes 7 , also shown after RBG-banding in the insert. 


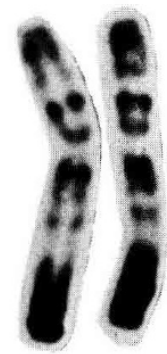

1

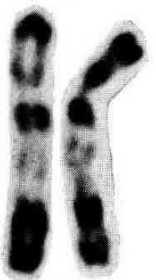

6

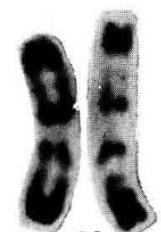

11

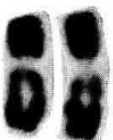

16

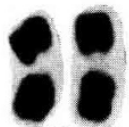

19

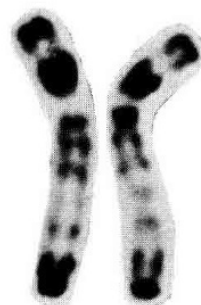

2

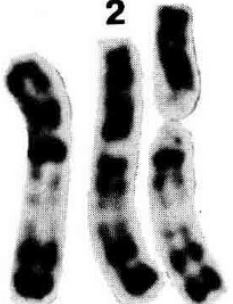

7

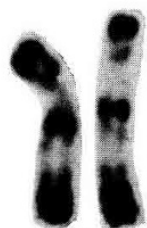

12

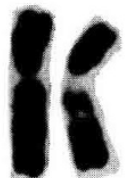

17

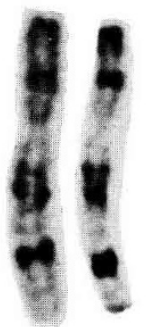

3

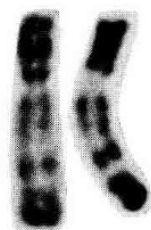

8

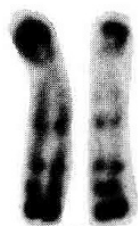

13

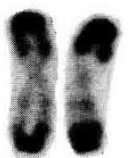

18

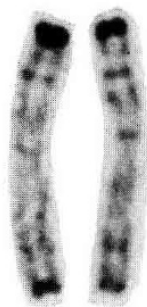

4

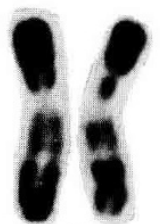

9

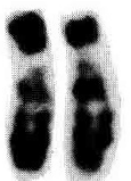

14

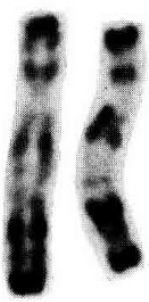

5

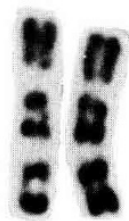

10

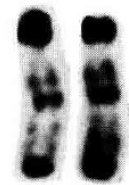

15

FIG, 2

Comparaison des chromosomes observés dans les genres Macaca, Papio et Lophocebus (à gauche) avec ceux de T. gelada (à droite). Une identité de marquage est retrouvée pour tous les chromosomes, en particulier pour les chromosomes 7 dans la forme non fissionnée.

Comparison of chromosomes observed in genera Macaca, Papio, and Lophocebus (left) with those of T.gelada (right). The chromosome banding is similar, particularly for non-fissionned chromosomes 7. 
cours de l'évolution. Cette absence s'oppose donc au fait que le seul remaniement intraspécifique connu soit une fission. Ceci peut recevoir deux explications :

- il existe une pression de mutations, entraînant des fissions, chez l'ensemble des Cercopithecidae (Cercopithecinae + Papioninae), mais celles-ci ne s'implantent que chez les Cercopithecinae pour des raisons liées à la structure des populations ou au mode de reproduction. En faveur de cette interprétation est le fait que l'évolution chromosomique, en général, est très pauvre chez les Papioninae, ce qui traduirait la difficulté d'implantation de tout remaniement chromosomique dans ce groupe. Cette hypothèse devrait toutefois être étendue à l'ensemble des Cercopithecoïdes, puisque le seul remaniement intraspécifique signalé chez un Colobidae est aussi une fission (EGOzCUE, 1971). Cependant, à l'inverse des Papioninae, les Colobidae ont subi une évolution chromosomique importante, mais sans qu'aucune fission ne soit survenue (DUTRILlaUX et al., 1982). Ce ne serait donc pas une difficulté d'implantation des remaniements chromosomiques en général, mais une sélection contre les fissions qu'il faudrait envisager ;

- les fissions observées à l'état hétérozygote chez un Papioninae et chez un Colobidae montreraient que ces groupes n'ont acquis qu'imparfaitement la possibilité de faire des fissions et de les transmettre, les Cercopithecinae ayant seuls achevé le processus. Cette hypothèse s'accorderait avec le fait que l'ensemble des Cercopithécoïdes possède un tronc commun. Au cours de cette évolution commune, plusieurs des caractères permettant les fissions auraient muté (mutations géniques, acquisition de séquences d'ADN répétitif, susceptibles de s'amplifier et de jouer le rôle de télomères et de centromères). Toutefois, la ou les dernières mutations nécessaires ne seraient survenues que dans la branche menant aux Cercopithecinae. Il est d'ailleurs intéressant de signaler que les fissions observées chez le $T$. gelada et chez le Colobidae (EgozcuE, 1971) sont centromériques, comme les rares cas dépistés chez l'homme, pour le chromosome 4 (Dallapiccola et al., 1976), le chromosome 7 (HANSEN, 1975 ; JANKE, 1982), le chromosome 10 (FrYNS et al., 1980) et un der $t$ (DqDq) (SINHA et al., 1972).

A l'opposé, la grande majorité des fissions survenues au cours de l'évolution des Cercopithecinae n'est pas centromérique et implique la formation d'un nouveau centromère au sein d'une structure chromatidienne.

Cette seconde hypothèse semble mieux s'accorder avec les données existantes, ce qui nous permet de proposer les conclusions suivantes :

- chez l'homme, seul primate étudié à grande échelle, les fissions sont exceptionnellement observées et toujours centromériques ;

- chez les Papioninae et les Colobidae, les fissions centromériques sont peut-être le remaniement intraspécifique le moins rare, puisqu'aucun autre remaniement n'est décrit dans ces groupes;

- chez les Cercopithecinae, les fissions deviennent efficaces sur le plan évolutif en même temps que leur mécanisme se perfectionne puisque les cassures ne sont plus nécesssairement centromériques.

Ce schéma hypothétique est donc en faveur de l'acquisition progressive de caractères qui vont orienter l'évolution chromosomique. Il s'intègre bien avec l'ensemble des observations faites sur les primates, puisque, comme nous l'avons déjà signalé (DuTRILLAUX, 1979 ; DUTRILlaUX et al., 1981) la distribution des remaniements dans l'arbre phylétique montre une très forte tendance à l'accumulation d'un type donné dans l'évolution d'un groupe donné d'animaux : translocations robertsoniennes chez les prosimiens, inversions péricentriques chez les Pongidae (TURLEAU et al., 1972), translocations chez les Hyloba- 
tidae (COUTURIER et et al., 1982). Ce phénomène semble même atteindre une très haute spécificité chez les Gerbillidae, où de multiples remaniements entre autosomes et chromosomes sexuels sont survenus (VIEGAS-PEQUIGNOT et al., 1982) alors que ceux-ci sont très rares par ailleurs, probablement parce qu'ils défavorisent la reproduction.

Signalons enfin que cette tendance, qui sous-entend le caractère non aléatoire de la mutagenèse chromosomique pourrait être très générale puisque WHITE l'avait décrite chez les insectes où il lui avait donné le nom d'orthoselection caryotypique (pour références, voir WHITE, 1978).

Reçu le 23 décembre 1982. Accepté le 24 janvier 1983.

\section{Remerciements}

Nous remercions Mme J. Mortier-Gabet pour son aide dans l'obtention des prélèvements. Aide financière : CNRS (ERA $n^{\circ} 47$ ), CEA (contrat B.C. 2334), EURATOM (contrat B10-E-398-F) et Electricité de France.

\section{Références bibliographiques}

Bernstein R., Pinto M., Morcom G., Bielert C., 1980. A reassessment of the karyotype of Papio ursinus. Homoeology between human chromosome 15 and 22 and a characteristic submetacentric baboon chromosome. Cytogenet. Cell Genet., 28, 55-63.

Cambefort Y., Mounie Ch., Colombies P., 1976 a. Topographies des bandes chromosomiques chez Papio papio. Ann. Génét., 19, 5-9.

Cambefort Y., Mounie Ch., Colombies P., 1976 b. Topographies des bandes chromosomiques chez Papio hamadryas. Ann. Génét., 19, 269-272.

Chiarelli B., 1962. Comparative morphometric analysis of primate chromosomes. II. The chromosomes of genera Macaca, Papio, Theropithecus and Cercocebus. Caryologia, 15, 401-420.

Cochet C., Creau-Goldberg N., Turleau C., Grouchy J. de, 1982. Gene mapping of Microcebus murinus (Lemuridae) : a comparison with man and Cebus capucinus (Cebidae). - Cytogenet. Cell Genet., 33, 213-221.

Couturier J., Dutrillaux B., Turleau C., Grouchy J. de, 1982. Comparaisons chromosomiques chez quatre espèces ou sous-espèces de gibbons. Ann. Génét., 25, 5-10.

Dallapiccola B., Mastroiacovo P., Gandini E., 1976. Centric fission of chromosome $n^{\circ} 4$ in the mother of two patients with trisomy 4p. Hum. Genet., 31, 121-125.

De Vries G.F., Geleijnse M.E.M., De France H.F., Hogendoorn A.M., 1975. Lymphocyte cultures of Macaca mulatta and Macaca fascicularis. Lab. anim. Sci., 25, 33-38.

Dutrillaux B., 1979. Chromosomal evolution in primates : tentative phylogeny from Microcebus murinus (Prosimian) to man. Hum. Genet., 48, 251-314.

Dutrillaux B., Viegas-Pequignot E., Dubos C., Masse R., 1978. Complete or almost complete analogy of chromosome banding between the baboon (Papio papio) and man. Hum. Genet., 43, 37-46.

Dutrillaux B., Biemont M.C., Viegas-Pequignot E., Laurent C., 1979 a. Comparison of the karyotypes of four Cercopithecoïdae : Papio papio, P.anubis, Macaca mulatta and M. fascicularis. Cytogenet. Cell Genet., 23, 77-83.

Dutrillaux B., Fosse A.M., Chauvier G., 1979 b. Étude cytogénétique de six espèces ou sousespèces de mangabeys (Papioninae, Cercopithecidae). Ann. Génét., 22, 88-92. 
Dutrillaux B., Couturier J., Viegas-Pequignot E., 1981. Chromosomal evolution in primates. Chromosomes today, M.D. Bennett, M. Bobrow, G. Hewirt eds., Allen and Unwin, London, 7, 176-191.

Dutrillaux B., Couturier J., Muleris M., Lombard M., Chauvier G., 1982. Chromosomal phylogeny of forty-two species or subspecies of cercopithecoids (Primates Catarrhini). Ann. Génét., 25, 96-109.

Egozcue J., 1971. A possible case of centric fission in a primate. Experientia, 27/8, 969-970.

Fryns J.P., Bulcke J., Hens L., VAN Den Berghe H., 1980. Balanced transmission of centromeric fission products in man. Hum. Genet., 54, 127-128.

HANSEN S., 1975. A case of centric fission in man. Humangenetik, 26, 257-259.

JANKE D., 1982. Centric fission of chromosome $n^{\circ} 7$ in three generations. Hum. Genet., 60, 200-201.

O'BRIEN S.J., NASH W.G., 1982. Genetic mapping in mammals : chromosome map of domestic cat. Science, 216, 257-265.

Sinha A.K., PathaK S., NORA I.J., 1972. A human family suggesting evidence for centric fission and stability of a telocentric chromosome. Human Heredity, 22, 423-429.

Soulie J., Grouchy J. de, 1981. A cytogenetic survey of 110 baboons (Papio cynocephalus). Am. J. Phys. anthropol., 56, 107-113.

Stock A.D., Hsu T.C., 1973. Evolutionary conservatism in arrangement of genetic material. A comparative analysis of chromosome banding between the Rhesus Macaque $(2 n=42,84$ arms $)$ and the African Green Monkey (2n=60, 120 arms). Chromosoma, 43, 211-224.

Turleau C., Grouchy J. de, Klein M., 1972. Phylogénie chromosomique de l'homme et des Primates hominiens (Pan troglodytes, Gorilla gorilla et Pongo pygmaeus). Essai de reconstitution de l'ancêtre commun. Ann. Génét., 15, 225-240.

Viegas-Pequignot E., Benazzou T., Dutrillaux B., Petter F., 1982. Complex evolution of sex chromosomes in Gerbillidae (Rodentia). Cytogenet. Cell Genet., 34, 158-167.

White M.J.D., 1978. Modes of speciation. Ed. W.H. Freeman and Cie, San Francisco. 